\title{
Influence of small cyclic and DC electrical loads on the fracture toughness of ferroelectric ceramics
}

\author{
Andrea R. Engert, Peter Neumeister ${ }^{\mathrm{a}}$, Matthias Mecklenburg ${ }^{\mathrm{b}}$, Hans Jelitto, \\ Herbert Balke ${ }^{\text {a }}$, Gerold A. Schneider \\ Technische Universität Hamburg-Harburg, Institut für Keramische Hochleistungswerkstoffe, Denickestrasse 15, 21073 Hamburg, Germany \\ ${ }^{a}$ Technische Universität Dresden, Institut für Festkörpermechanik, Mommsenstrasse 13, 01062 Dresden, Germany \\ ${ }^{b}$ Technische Universität Hamburg-Harburg, Institut für Kunststoffe und Verbundwerkstoffe, Denickestrasse 15, 21073 Hamburg, Germany
}

Received 16 July 2010; received in revised form 25 October 2010; accepted 7 November 2010. Available online 8 December 2010

Corresponding author. Tel.: +49 40 42878-3037, E-Mail: g.schneider@tu-harburg.de. Homepage: www.tu-harburg.de/gk

\begin{abstract}
The present work investigates the influence of small AC, unipolar cyclic and DC electric loads on the fracture toughness of a ferroelectric ceramic. Single-edge-notched beams (SENB) of a poled and unpoled lead zirconate titanate (PZT) ceramic were fractured in a four-point-bending device under conditions of controlled crack growth. During crack advance the different electrical loads were applied perpendicular to the crack faces. The applied nominal electric field amplitudes were less than one third of the coercive field and maximum frequencies of $20 \mathrm{kHz}$ were applied. The measured R-curves show that even for these low amplitudes, electrical AC loading causes a drop in the critical mechanical load of up to $25 \%$. The drop increases with increasing amplitude and frequency. Possible mechanisms to explain the results are discussed.
\end{abstract}

Keywords: Fatigue; Ferroelectric properties; Fracture; PZT; AC, unipolar cyclic and DC electrical loading

\section{Introduction}

Ferroelectric ceramics are used, amongst other applications, in electro-mechanical transducers, converting mechanical forces into an electric potential (direct piezoelectric effect) or vice versa (inverse piezoelectric effect). They offer advantages such as nanometer precision in positioning, large force generation and fast response time in actuator applications (electro-mechanical transducer), high sensitivity as force or displacement sensors (mechanoelectrical transducer), and operation in a high frequency range as ultrasonic transmitters or receivers [1]. During their lifetime, ferroelectric ceramics must be able to operate under long-term cyclic electro-mechanical loading. While they continue to find increasing use, questions regarding lifetime and reliability are still investigated due to their low fracture toughness and complex material behaviour. This work seeks to add to the understanding of fracture under combined quasi static mechanical and constant or cyclic electrical loading. In the following a short introduction and literature review is given about mechanical and electrical DC- and AC-loading of cracks in ferroelectric ceramics. Afterwards the motivation and the contents of this work are introduced.

\subsection{Ferroelectric $R$-curve behaviour}

Ferroelectric ceramics have been shown to exhibit crack growth resistance (R-curve) behaviour [2-,6], i.e. a rising fracture toughness vs. crack length. The toughening mechanism has been predominantly attributed to domain switching [7-9]. The received opinion about this mechanism is described e.g. in a review by Schneider [10], and can be summarized as follows: in the case of tensile loading, the stress concentration at a crack tip leads to ferroelastic switching, in which the domains switch to align with the load such that no remanent polarization, but a remanent strain, is induced. Once the crack tip advances into the switched zone, a local compressive residual stress state is created in the vicinity of the crack. This residual stress results from the misfit between the switched region and the bulk material and shields the advancing crack tip from the applied mechanical load. Consequently a larger load is required for the crack to continue propagating. In case of small-scale switching, where the zone of active switching is much smaller than any other geometric lengths, a steady state toughness, i.e. a plateau in the R-curve, is reached when (i) the zone of active switching holds a constant size and when (ii) the zone behind the tip that contributes to shielding, has a sufficient length. Fett et al. [11] compared R-curves from compact tension (CT) and single-edge-notched beam (SENB) specimens, 
showing that in 4-point flexure the values for the fracture toughness are higher than for CT tests, and that a plateau value is not reached.

\subsection{Influence of DC electrical load on fracture toughness}

A crack filled with air in a ferroelectric material is a void with a relative dielectric constant of 1 in a matrix with a dielectric constant that is typically three orders of magnitude higher. If an electric field is applied normal to the crack surface, it will penetrate the crack. The crack shape, the crack opening displacement and the ratio of the permittivities of the crack interior and the ceramic, will determine to what extent the electric flux through the crack will be diluted, and increased ahead of the tip. For cracks in a ferroelectric ceramic, i.e. in an electromechanically coupled material, this leads to a non-linear boundary value problem.

To describe cracks in ferroelectric materials, four crack models are used in the literature: (i) impermeable (relative dielectric constant of the crack equal to zero), (ii) permeable (potential drop across the crack equal to zero), (iii) semipermeable or exact (relative dielectric constant of the crack equal to one) [12-14] and (iv) energetically consistent (relative dielectric constant of the crack equal to one, electrostatic tractions along the crack faces) $[15,16]$. Li et al. [17] calculated the mechanical mode I stress intensity factor $K_{\mathrm{I}}$ and the energy release rate (energy release per crack advance) for a stationary crack in a SENB-specimen loaded with a constant mechanical force. They compared, for the different crack models, the effect of an additional electric field applied perpendicular to the crack faces on those two parameters. The impermeable crack model does not predict any influence of the electrical load on $K_{\mathrm{I}}$, but it predicts a reduction of the energy release rate with increasing electrical load. Together with an energy-based crack growth criterion the latter suggests increasing critical mechanical loads with increasing electric field. The energetically consistent model predicts that under an electric field both $K_{\mathrm{I}}$ and the energy release rate are reduced. The permeable crack model does not predict any influence of an applied electrical load neither on $K_{\mathrm{I}}$ nor on the energy release rate. Several experiments have been performed with constant electrical load to validate the theoretical predictions. E.g. R-curves under electromechanical loading were measured for SENB-PZTspecimens by Jelitto et al. [18,19] and for SENB-PZTactuators by Häusler et al. [20]. Both did not observe a decisive change of the critical mechanical loads as a consequence of a DC electric field of one third of the coercive field $E_{\mathrm{c}}$. Furthermore, Schneider et al. [21] applied an electrical load perpendicular to a crack induced by a Vickers indent in PZT. They measured the crack opening together with the drop of the electric potential across the crack. From these data they determined an effective relative permittivity of the crack of 40 , and showed that the crack tip energy release rate calculated for such a crack is almost equal to the one of a permeable crack. All these experimental observations argue for the permeable crack model. Nevertheless one should take into account that an electric field concentration ahead of the crack tip is likely to occur.

\subsection{Crack growth due to AC voltage}

Experiments have shown that cracks in ferroelectric ceramics can be driven by an AC voltage applied perpendicular to the crack faces. The first fatigue crack growth experiments were observed for cracks introduced by Vickers indents [22-25]. Cao and Evans [23] assumed domain switching at the crack tip and at contacting crack bridges leading to a strain mismatch which causes the crack to grow. Lynch et al. [24] measured the crack extension in PZT against the cycle number for electric fields between $1.1 E_{\mathrm{c}}$ and $1.7 E_{\mathrm{c}}$ and using frequencies of $1.5 \mathrm{~Hz}$ and $5 \mathrm{~Hz}$. They found higher crack growth rates for higher fields. No influence of the frequency was observed. To explain the mechanism for crack advance, they introduced a globalmismatch model based on the ferroelectric strain hysteresis. Shieh et al. [26] performed experiments on through-thickness cracks in PZT with AC fields of $20 \mathrm{~Hz}$ and amplitudes of 0.9 $E_{\mathrm{c}}$ to $3.0 E_{\mathrm{c}}$. They found fine material debris resulting from abrasive wear on the fracture surfaces and microcracking. Investigation of fatigue crack growth under pure $A C$ and combined AC electromechanical loading in PZT-DCB (double cantilever beam) samples were done by Westram et al. [27,28]. They used electrical loads of $1 \mathrm{~Hz}$, amplitudes of $0.9 E_{\mathrm{c}}$ to $1.9 E_{c}$, and additional constant mechanical loads that correspond to a mode I stress intensity factor of $0.1 \mathrm{MPa} \sqrt{\mathrm{m}}$ to $0.5 \mathrm{MPa} \sqrt{\mathrm{m}}$. The cracks advanced during every field reversal when the coercive field was approached and arrested before the maximum field was reached. To explain the observations a finite element model based on large-scale domain switching was used, in which a semipermeable crack with a dielectric constant of 1 was assumed [27]. In the experiments with electromechanical loading, crack growth rates increased with the additional mechanical load [28]. Nam et al. [29] applied AC, uni- and sesquipolar (sesqui (lat.): one-and-one-half times) cyclic electric fields of $5 \mathrm{~Hz}$ and of amplitudes of $0.8 E_{\mathrm{c}}$ to $1.3 E_{\mathrm{c}}$ to DCB samples. For the unipolar and sesquipolar loading, crack growth rates were almost negligible compared to AC loading.

\subsection{Motivation and contents}

The works described above focus on fatigue crack growth induced by nominal cycling electric fields with magnitudes in the order of $E_{\mathrm{c}}$ or higher; constant mechanical loads are used as secondary loads; crack growth rates are recorded vs. cycle numbers. We, on the other hand, investigate the influence of small AC, unipolar cyclic and DE electric fields far below $E_{\mathrm{c}}$ on the critical mechanical loads. Homogeneous bars made of poled and unpoled PZT ceramic are cracked under controlled crack growth in a four-point-bending set-up. In addition to the mechanical load the different electrical loads with nominal field amplitudes between 0 (no electric field) and $350 \mathrm{~V} / \mathrm{mm}\left(<0.4 E_{\mathrm{c}}\right)$ and frequencies between 0 (DC) and $20 \mathrm{kHz}$ are applied perpendicular to the crack. To evaluate the effect of the electrical loads on the critical crack tip loading, the change of the critical mechanical load is related to crack tip load parameters. Finally, the influence of frequency and amplitude of AC electrical loads is studied and the effect of AC loads is compared with the one of unipolar cyclic and DC loads. 


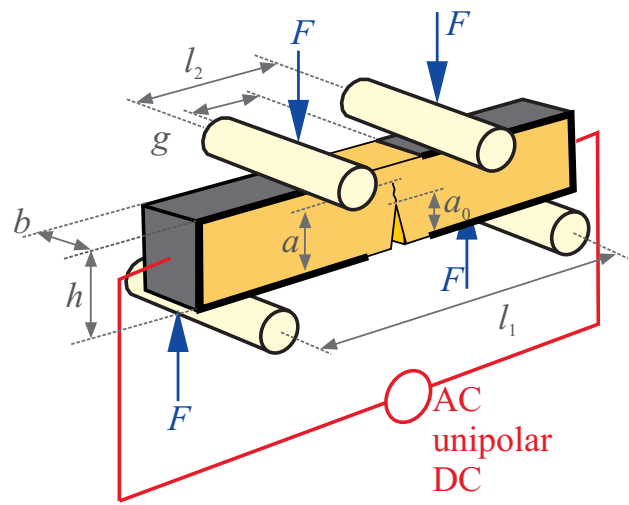

Figure 1 Schematic drawing of a SENB-specimen with a straight notch through the specimen; force and electrical load application via rollers and U-shaped electrodes $(\mathrm{b} \approx 3 \mathrm{~mm}, \mathrm{~h} \approx 4 \mathrm{~mm}$, $\mathrm{a}_{0} \approx 1 \mathrm{~mm}, \mathrm{~g} \approx 2 \mathrm{~mm}, \mathrm{l}_{1}=20 \mathrm{~mm}, \mathrm{l}_{2}=10 \mathrm{~mm}$ ).

\section{Experiments}

\subsection{Material and specimen preparation}

The experiments were performed with bars made of a commercial soft PZT (PIC151, PI Ceramic GmbH, Germany) [1,30,31]. Poled and unpoled samples from the same powder batch were used. The poled samples had been poled at room temperature with an electric field of $2 \mathrm{kV} / \mathrm{mm}$ parallel to the bar axis by the supplier. According to the data sheet the relative dielectric constant $\varepsilon_{33}$ of the poled material in poling direction is 2400 [32]. The material's grain size specified by the supplier is $6 \mu \mathrm{m}$. The coercive field $E_{\mathrm{c}}$ read from a hysteresis measurement (room temperature, $2 \mathrm{kV} / \mathrm{mm}$ maximum field, $5 \mathrm{mHz}$ ) is $1 \mathrm{kV} / \mathrm{mm}$.

A schematic drawing and a photo of a sample are shown in Figure 1. All bars had approximately a width $b$ of $3 \mathrm{~mm}$, a height $h$ of $4 \mathrm{~mm}$ and a length $l$ of $27 \mathrm{~mm}$. The front face $(4 \mathrm{~mm} \times 27 \mathrm{~mm})$ was polished down to a grain size of $1 \mu \mathrm{m}$. An oxide polishing step with an acidic alumina suspension (OP-A on MD-Chem, Struers) was used to reveal the grain boundaries. Then a notch was cut in the face oriented for tension with a cutting disk. The notches were then extended to a length $a_{0}$ of around $1 \mathrm{~mm}$ and at the same time sharpened using a razor blade with diamond paste $[33,34]$. The final notches had tip radii $r$ of around $10 \mu \mathrm{m}$. Silver electrodes were painted onto the ends as well as onto the top and bottom faces ( $3 \mathrm{~mm} \times 27 \mathrm{~mm}$ faces) of the bar, leaving a gap $g$ of approximately $2 \mathrm{~mm}$ symmetrically around the notch. For every sample, the width and the height were determined by a slide calliper, the notch length and the gap between the electrodes were determined through images taken with an optical polarizing microscope.

\subsection{Instrumentation and measuring procedure}

The R-curve measurements were performed with a custom made, semi-automatic four-point-bending device [35]. Nonconducting $\mathrm{Al}_{2} \mathrm{O}_{3}$ rollers with a diameter of $5 \mathrm{~mm}$ and roller distances of $l_{1}=20 \mathrm{~mm}$ and $l_{2}=10 \mathrm{~mm}$ were used. The force $F$ was measured with a quartz compression high impedance load cell (9212, Kistler). The data points were captured in the following sequence: The force was slowly increased until the crack was observed to propagate. At this instance the force was reduced by $\approx 20 \%$. This is further called partial unloading. The crack length $a$ was read through a stereo microscope and entered into the computer program, which also recorded the maximum or critical force $F_{\mathrm{c}}$. The time needed to record one data point was around 10 seconds. This speed of measuring was similar for all measurements. Since the quartz force sensor was known to drift, the samples were completely unloaded at several points during the measurements. The force readings at these complete unloadings were used to correct for the drift using a polynomial of $2^{\text {nd }}$ or $3^{\text {rd }}$ order depending on the drift.

A low voltage DC, AC or unipolar signal was produced by a DC voltage generator or a function generator respectively. The low voltage signal was then amplified by a high-voltage DC-stable piezo amplifier with a voltage range of 0 to $\pm 700 \mathrm{~V}$ and a large signal bandwidth of $15 \mathrm{kHz}$ (PZD700, TREK). The frequency $f$, the amplitude $U_{0}$ and the DC bias $U_{1}$ of the output voltage $U(t)$

$$
U(t)=U_{0} \sin (2 \pi f t)+U_{1},
$$

were verified with an oscilloscope. Due to the measuring speed of one data point per $10 \mathrm{~s}$, the crack was subjected to more AC loading cycles, when an $\mathrm{AC}$ voltage with a higher frequency was applied.

In order to determine the effect of the electrical load on the critical mechanical load, each sample was cracked piecewise, in turn with purely mechanical and combined mechanical and electrical load. I.e. the crack was initiated from the notch by mechanical loading. After around 10 data points were captured, the additional electrical load was turned on; again 10 data points were captured, before the electrical load was turned off, etc. Each such R-curve "piece" with constant type of loading is referred to as section.

\subsection{Tested samples}

A total of 18 poled samples were used to measure R-curves with combined mechanically static and AC electrical load (AC electromechanical loading, $\mathrm{m}+\mathrm{AC}$ ). Eight samples were measured with a constant amplitude of $700 \mathrm{~V}$ applied in every AC section of the R-curves. Another ten samples were cracked with varying amplitude of the applied voltage from on electromechanical section to the next. Table 1 gives a list of the amplitudes and frequencies of the electrical load applied to each sample.

Additionally to the AC measurements the experiment was modified in four different ways: firstly, the same type of measurement was done with unipolar cyclic loading in ( 0 to $+700 \mathrm{~V})$ and opposite to $(0$ to $-700 \mathrm{~V})$ the poling direction with frequencies of $100 \mathrm{~Hz}$ and $1 \mathrm{kHz}$ (unipolar electromechanical loading, $\mathrm{m}+$ uni \pm ). Secondly a DC load was applied in $(+700 \mathrm{~V})$ and against $(-700 \mathrm{~V})$ the poling direction (DC electromechanical loading, $\mathrm{m}+\mathrm{DC} \pm$ ). Thirdly, only one AC loading cycle $(1 \mathrm{~Hz}, \pm 700 \mathrm{~V})$ was applied when the specimen was partially unloaded, i.e. while the crack was stopped. Then the specimen was again reloaded mechanically until the crack propagated. In another measurement the sample was loaded for $1 \mathrm{~s}$ with $1 \mathrm{kHz}\left(10^{3}\right.$ cycles, $\left.\pm 700 \mathrm{~V}\right)$ during the partial unloading when the crack was stopped. And as a last modification, an $\mathrm{AC}$ load of $100 \mathrm{~Hz}$ and $1 \mathrm{kHz}$ $( \pm 700 \mathrm{~V})$ was applied to unpoled samples. 


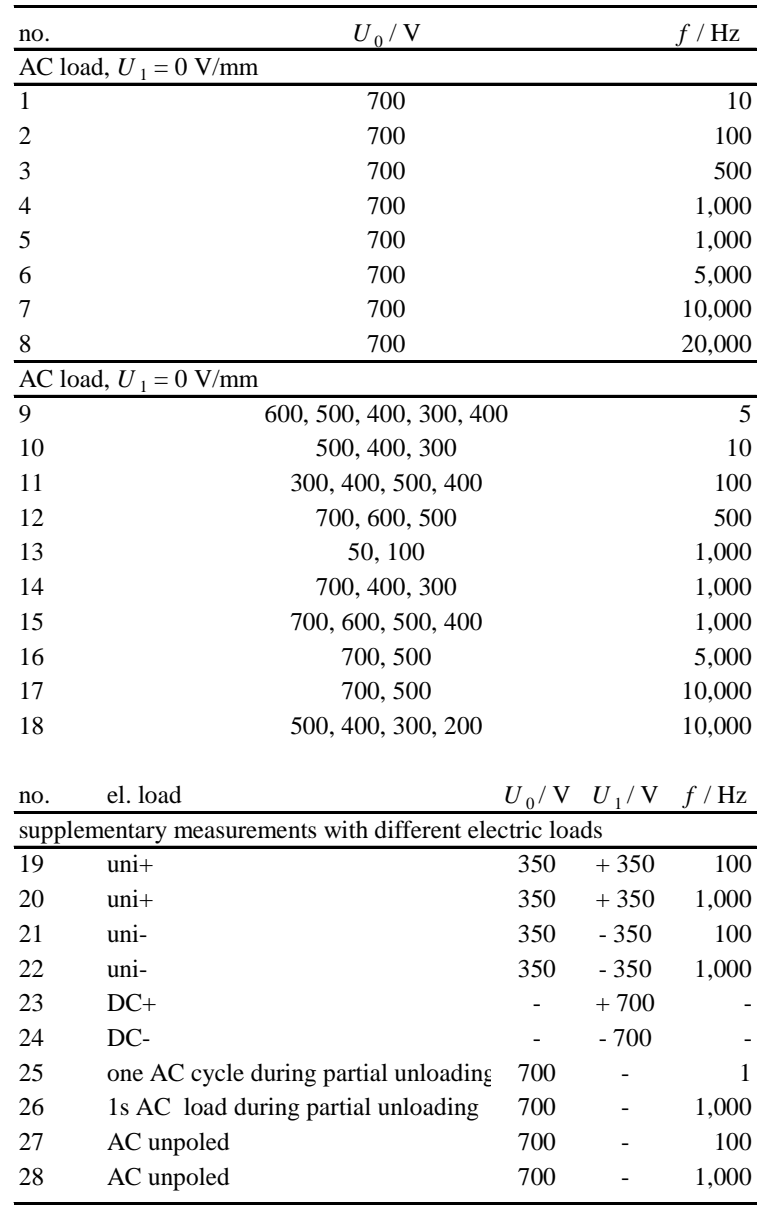

Table 1 List of all samples used for AC and supplementary measurements. + and - denote electrical loading in and against the poling direction.

Two further samples were used to make images of the crack path and the fracture surface. For the images of the crack path, a sample was cracked under pure mechanical load half way through, so that the sample was still in one piece in the end. For the images of the fracture surface, a second sample was loaded purely mechanically until the crack was half way through the sample, and then loaded with an additional AC load of $700 \mathrm{~V}$ amplitude and $100 \mathrm{~Hz}$ frequency in the second partway.

\section{Results}

\subsection{Determination of the crack tip load and the relative drop in the fracture toughness}

\section{Mechanical and electrical crack tip load}

In order to evaluate the effect of the AC loading on the critical mechanical load independently of the sample geometry and the crack length, the measured critical force $F_{\mathrm{c}}$ and the amplitude of the applied AC load $U_{0}$ at a specific relative crack length $a / h$ have to be related to crack tip load parameters. Due to the non-uniform electric field distribution in the sample, the formulation of such independent parameters is not straightforward. In the calculations the crack is considered to be electrically permeable.
In linear elastic fracture mechanics $F_{\mathrm{c}}$ is related to the fracture toughness $K_{\mathrm{IC}}$. In ferroelectric ceramics this is only possible for small-scale switching, i.e., if the zone of ferroelastic domain switching around the crack tip is small compared to any other characteristic length of the cracked specimen. Jelitto et al. [35] estimated the radius of the switching zone of a statically driven crack in a SENBspecimen made of PZT PIC151 to $0.15 \mathrm{~mm}$. Hence, the condition of small-switching are satisfied if a $>0.15 \mathrm{~mm}$ for short cracks or $(\mathrm{h}-\mathrm{a}) \gg 0.15 \mathrm{~mm}$ for long cracks. This is true for $1 \mathrm{~mm}<a<3 \mathrm{~mm}$ or with a sample height of $4 \mathrm{~mm}$ for $25 \%<a / h<75 \%$. In case of poled samples, the nonuniform electric field might contribute to the mode I crack tip load significantly. With the help of an FE analysis presented in Appendix A, it was possible to show that this effect is negligible. Hence, $K_{\mathrm{I}}$ is caused by the mechanical load only. $K_{\mathrm{I}}$ was computed with the help of a shape function for SENB-specimens given by Munz and Fett [36, p. 279] (Appendix B). To account for a rising fracture toughness, the critical mode I stress intensity factor $K_{\mathrm{IC}}$ is named $K_{\mathrm{R}}$.

In order to characterize the effect of the electrical load on the crack tip, the nominal electric field $E_{\text {nom }}$ at the current crack tip position $a$ is introduced. It is derived with the help of a FE analysis (Appendix C). The use of $E_{\text {nom }}$ is justified by the assumption that the mechanisms responsible for the effect of the electrical loading occur only in a small region in the vicinity of the crack tip so that the change of $E_{\text {nom }}$ within this region can be neglected.

\section{Relative drop in the fracture toughness}

Figure 2 shows the R-curve of a measurement where the applied AC voltage has an amplitude of $700 \mathrm{~V}$ and a frequency of $100 \mathrm{~Hz}$. (sample 2 in Table 1). The crack was initiated by mechanical loading $(\mathrm{m})$. When the additional AC load was turned on $(m+A C)$, the critical mechanical force to drive the crack decreased. This leads to a drop in the calculated fracture toughness. Several difficulties were encountered in the determination of $\Delta K_{\mathrm{el}}$ : (i) the scattering of $K_{\mathrm{R}}$ within one section, (ii) the fact that $K_{\mathrm{R}}$ is measured either for purely mechanical load or combined electromechanical load but never for both at the same time (i.e. at the same crack length), and (iii) when the specimen was reloaded after a complete unloading the fracture toughness was first lower than it had been before unloading, and rose to a steady state value within a few loading cycles. To even the scattering and to extrapolate the R-curves sections both R-curve branches, with and without electrical loading, were fitted separately with a polynomial of forth, fifth or sixth order. The degree of the polynomial was chosen depending on the number of electrically loaded sequences and depending on the ability not only to follow the data points but also to give a reasonable connection of the sections. The data points after the complete unloadings, which were not used for the fit, were determined for every section individually and excluded from the fit. In the end $\Delta k_{\mathrm{el}}$ was determined for one discrete relative crack length in the middle of each electromechanically loaded section using the two fit curves (inserted graph in Figure 2). Thus, one discrete value of $\Delta k_{\mathrm{el}}$ was derived for each electromechanically loaded section. 


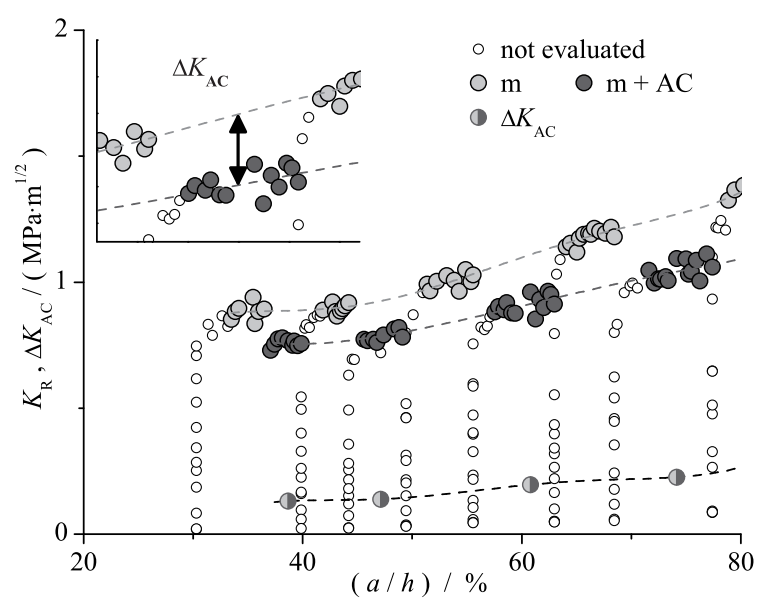

Figure 2 R-curve (critical stress intensity factor vs. the relative crack length) with piecewise pure mechanical $(\mathrm{m})$ and combined mechanically static and AC electrical load $(m+A C)$ with $100 \mathrm{~Hz}$ and $700 \mathrm{~V}$ amplitude. The graph also shows $\Delta \mathrm{K}_{\mathrm{AC}}$ as the distance of the two fitted curves. The dashed lines are the polynomial fits. The inserted graph illustrates the determination of $\Delta \mathrm{K}_{\mathrm{AC}}$ for one $\mathrm{AC}$ loaded R-curve section.

\subsection{Fracture toughness reduction due different types of electrical loading}

\section{Effect of AC loading on the fracture toughness}

To visualize the effect of amplitude and frequency of the AC load on the fracture toughness, $\Delta k_{\mathrm{AC}}$ is plotted vs. the nominal electric field amplitude $E_{\text {nom }}$ at the crack tip and the frequency $f$ (Figure 3 ). A logarithmic scale (lg - decadic logarithm) is chosen for the frequency. The data points for this 3D-plot are taken only from the AC measurements with poled samples (samples 1 to 18 in Table 1) for crack lengths in the range of $25 \%<a / h<75 \%$ (see Section 3.1). E.g. the R-curve shown in Figure 2 contributed four $\Delta k_{\mathrm{AC}}\left(E_{\mathrm{nom}}, 100 \mathrm{~Hz}\right)$ triples to the 3D-plot. $\Delta k_{\mathrm{AC}}$ is found to increase with increasing nominal amplitude and frequency of $E_{\text {nom. }}$. To express the effect of the electrical load, taking into account the non-congruent R-curves of different samples, a relative drop in the fracture toughness due to an electrical load $\Delta k_{\mathrm{el}}$ is defined as:

$$
\Delta k_{\mathrm{el}}(a)=\frac{\Delta K_{\mathrm{el}}(a)}{K_{\mathrm{m}}(a)}=\left(\frac{K_{\mathrm{m}}(a)-K_{\mathrm{m}+\mathrm{el}}(a)}{K_{\mathrm{m}}(a)}\right) .
$$

$K_{m}$ and $K_{m+e l}$ denote the fracture toughness under pure mechanical and under combined electromechanical loading, respectively. In the experiments $\mathrm{AC}$, unipolar and $\mathrm{DC}$ electrical loads were used. In order to distinguish the different types of electrical loading the index ${ }_{m+e l}$ is replaced by $\mathrm{m}+\mathrm{AC}$ for $\mathrm{AC}$ electromechanical loading and $\mathrm{m}+$ uni \pm and $\mathrm{m}+\mathrm{DC} \pm$ for unipolar and DC electromechanical loading in (+) and against (-) the poling direction. $\Delta k_{\mathrm{el}}>0$ corresponds to a decrease of the fracture toughness as a consequence of an additional electrical load. In order to derive an empiric relation, $\Delta k_{\mathrm{AC}}$ was investigated along (a) a plane of constant frequency and (b) a plane of constant amplitude, respectively. Hereby only data points measured at a relative

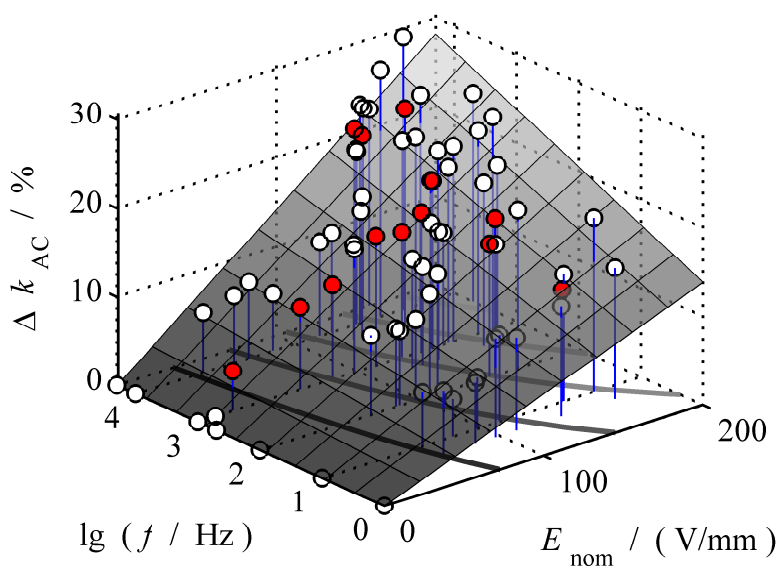

Figure 3 3D plot of the normalized drop in the fracture toughness during AC electromechanical loading vs. the frequency and nominal electric field amplitude at the crack tip - measured data and bilinear fit. The data points with a relative crack length between $45 \%$ and $65 \%$, and a nominal field amplitude of $(150 \pm 10) \mathrm{V} / \mathrm{mm}$ or a frequency of $1 \mathrm{kHz}$, which were used separate linear fits, are dark coloured.

crack length between $45 \%$ and $65 \%$ were used. For the first plane a frequency of $1 \mathrm{kHz}$ was chosen (Figure $4 \mathrm{a}$ ). For the second one points with a nominal electric field of $(150 \pm 10) \mathrm{V} / \mathrm{mm}$ were selected (Figure $4 \mathrm{~b}$ ). Both plots show a linear dependency of $\Delta k_{\mathrm{AC}}$ on $E_{\mathrm{nom}}$ and $\lg (f / \mathrm{Hz})$. Consequently, a bilinear fit was used to quantify $\Delta k_{\mathrm{AC}}$ against $E_{\text {nom }}$ and $\lg (f / \mathrm{Hz})$. Together with the reasonable assumption that $\Delta k_{\mathrm{AC}}\left(E_{\mathrm{nom}}=0 \mathrm{~V} / \mathrm{mm}\right)=0$ the fit function takes the general form:

$$
\Delta k_{\mathrm{AC}} / \%=\left(\mathrm{m}_{1}+\mathrm{m}_{2} \lg (f / \mathrm{Hz})\right) E_{\text {nom }}
$$

$\mathrm{m}_{1}$ was derived from the intercept with the ordinate of the 2D fit presented in Figure $4 \mathrm{~b}$ divided by $150 \mathrm{~V} / \mathrm{mm}$ to $\mathrm{m}_{1}=10.34 /(150 \mathrm{~V} / \mathrm{mm})=0.069 \mathrm{~mm} / \mathrm{V} . \mathrm{m}_{2}$ had to be fitted to the 3D-data directly. A value of $\mathrm{m}_{2}=0.017 \mathrm{~mm} / \mathrm{V}$ was found. Figure 3 shows the empiric fit in comparison to the measured data.

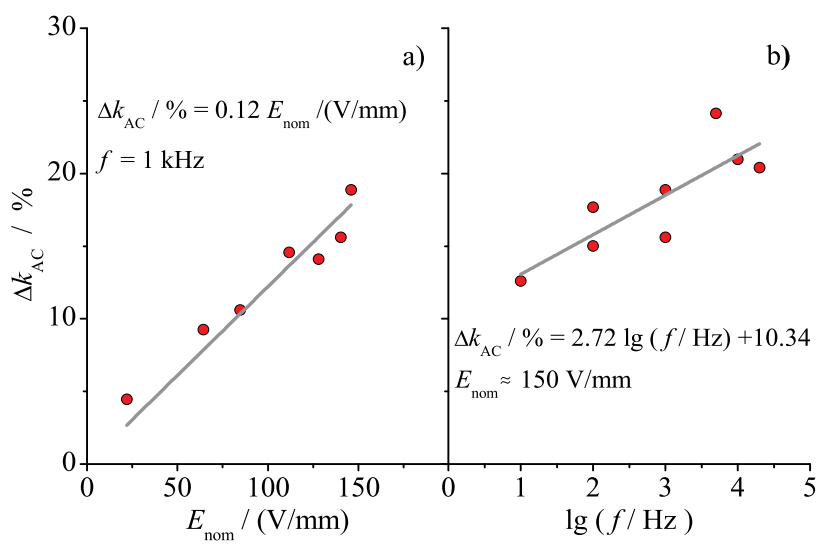

Figure 4 Relative drop in the fracture toughness during $\mathrm{AC}$ electromechanical loading for a relative crack length between $45 \%$ and $65 \%$ : (a) vs. the nominal electric field amplitude at the crack tip, for a constant frequency of $1 \mathrm{kHz}$ and (b) vs. the frequency, for a nominal electric field amplitude at the crack tip of $(150 \pm 10) \mathrm{V} / \mathrm{mm}$. 


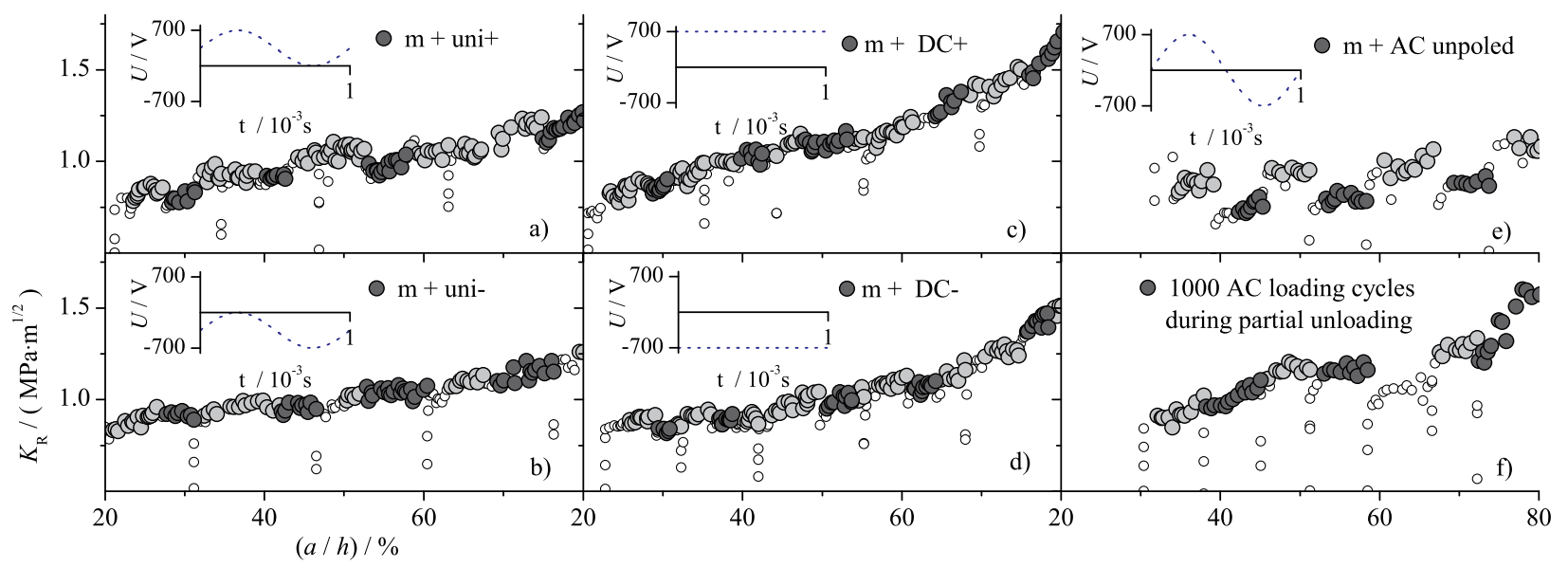

Figure 5 R-curves (critical stress intensity factor over the relative crack length) showing the effect of the different additional electrical loads: (a) unipolar load in poling direction, $100 \mathrm{~Hz}, 350 \mathrm{~V}$ (sample 19), (b) unipolar load against poling direction, $100 \mathrm{~Hz}, 350 \mathrm{~V}$ (sample 21), (c) DC load in poling direction, +700 V (sample 23), (d) DC load against poling direction, -700 V (sample 24), (e) unpoled sample, AC load, $1 \mathrm{kHz}, 700 \mathrm{~V}$ (sample 27). (f) 1,000 AC loading cycles during partial unloading, $1 \mathrm{~s}, 1 \mathrm{kHz}, 700 \mathrm{~V}$ (sample 26). The Rcurve sections marked with "test" were made with a (permanent) AC load during crack growth $(1 \mathrm{kHz}, 700 \mathrm{~V})$ to check the electrical set-up.

\section{Effect of unipolar and DC electrical loads on the fracture toughness}

Figure 5 shows some exemplary R-curves obtained from the supplementary measurements of samples 19 to 28 given in Table 1. The impact of the used loads on the fracture toughness can be summarized as follows:

- unipolar in poling direction

- unipolar against poling

$$
\begin{array}{ll}
\Delta k_{\text {uni+ }} & <4 \% \\
\Delta k_{\text {uni- }} & \approx 0 \\
\Delta k_{\mathrm{DC}+} & \approx 0 \\
\Delta k_{\mathrm{DC}-} & <2 \% \\
\Delta k_{\mathrm{AC}, \text { unloaded }} & \approx 0 \\
\Delta k_{\mathrm{AC}, \text { unpoled }} & >20 \%
\end{array}
$$

- DC in poling direction

- DC against poling direction

- AC during partial unloading$$
\approx \Delta k_{\mathrm{AC}}
$$

\subsection{Crack path and fracture surface}

The images taken with an optical microscope in Figure 6 show the crack path of a mechanically driven crack. They indicate e.g. crack deflection, crack bridging and predominantly intergranular crack growth.

The polished surface is believed to show the true microstructure on the scale of the grains, i.e. the dark spots are recognized as pores and not as pull-outs. The pictures confirm the grain size of $6 \mu \mathrm{m}$ specified by the supplier.A series of SEM pictures was taken throughout the whole fracture surface of the sample that was cracked under pure mechanical load in the first half and with combined AC electromechanical loading in the second half of static crack elongation. The exemplary images in Figure 7 do not show a difference between the fracture surface for the pure mechanical loading (Figure 7a) and the combined electromechanical loading (Figure 7b). Both pictures show a combination of intergranular and transgranular fracture.

\section{Discussion}

The R-curve in Figure 2 shows that once the additional AC load is turned off, the fracture toughness increases by the same amount, by which it had decreased when the AC load was turned on. Although we do not know, how the R-curve would look like for pure mechanical loading, i.e. without the electromechanically loaded sections, we believe that it would be congruent with the mechanically loaded sections. This would indicate that there is neither a destruction of the ferroelastic switching zone nor a damage of the bulk material.

In the $3 \mathrm{D}$ plot, the drop in the fracture toughness increases with the nominal electric field amplitude and with the frequency. The increasing drop in the fracture toughness with the amplitude could indicate higher stresses and strains in front of the crack tip. The fact that $\Delta k_{\mathrm{AC}}$ also increases with the frequency could be explained by the fact that at a higher frequency more loading cycles are applied to the crack.

a)

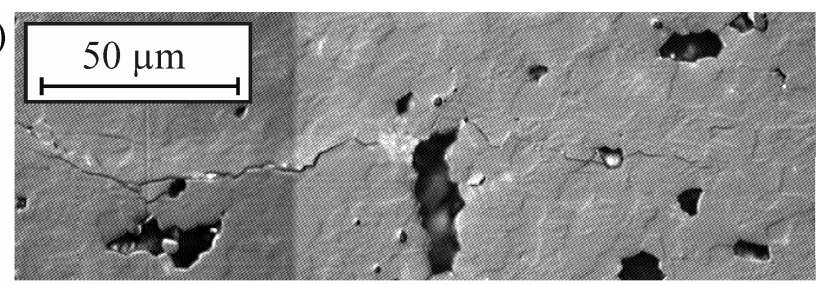

b)

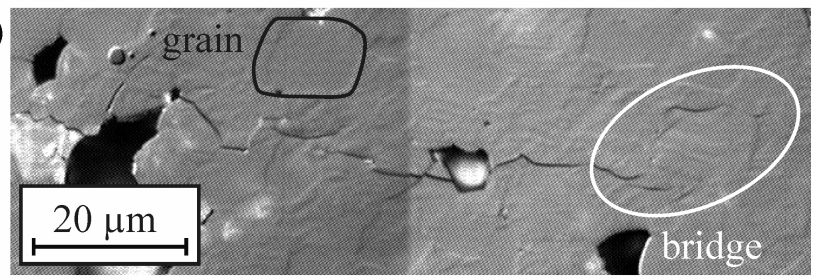

Figure 6 Optical microscope images of the crack path of a mechanically driven crack in two different magnifications a) and b), showing intergranular crack growth, crack deflection, crack bridging and the microstructure with a grain size of about $6 \mu \mathrm{m}$. 
a)

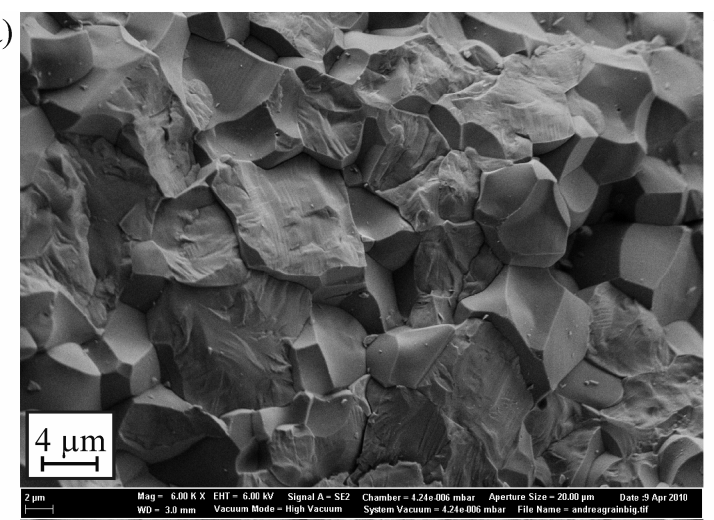

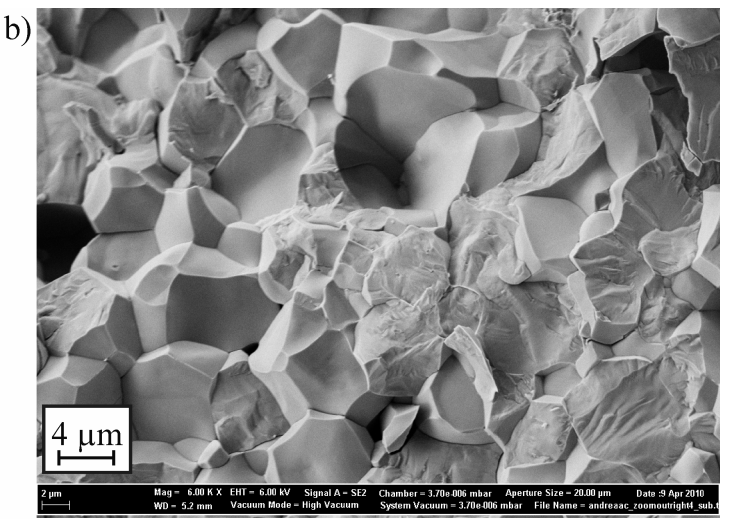

Figure 7 SEM images of a fracture surface showing (a) the fracture surface created during pure mechanical and (b) combined AC electromechanical loading. The pictures show a combination of intergranular and transgranular fracture.

The R-curves with unipolar cyclic and DC loading show that the microscopic events leading to a distinctive lower fracture toughness are only caused by AC loads, independently of the state of poling. The fact that $\Delta k_{\mathrm{AC}}$ is negligibly small for unipolar loading argues for domain switching with polarization reversal being involved in the drop of the fracture toughness. From the measurements of the unpoled samples it can be confirmed, that the drop in the fracture toughness for poled samples is not caused by a macroscopic effect of the U-shaped electrodes. When the AC voltage was applied during partial unloading of the crack, i.e. when it was stopped, the fracture toughness was not reduced when the crack was further propagated. This means, that the AC load does not lead to a damage of the material in front of the crack tip - if it is applied when the crack is closed.

\section{Potential crack growth mechanism}

The evaluation of the experimental results is based on two assumptions (see Section 3.1): (i) no effect of the U-shaped electrodes on $K_{\mathrm{I}}$ and (ii) the permeable crack model. The former is justified by both the $\mathrm{FE}$ analysis presented in Appendix A and the experimental finding that the AC effect appears with the same magnitude also for unpoled samples. Concerning the permeable crack model, the DC experiments show clearly that, as expected for a permeable crack, the effect of the applied electric field on $K_{\mathrm{R}}$ is negligible for DC electromechanical loading of around $1 / 3 E_{\mathrm{c}}$. Therefore, it can be confirmed that the permeable crack model is a reasonable assumption for the DC measurements. However, it can not explain the drop in $K_{\mathrm{R}}$ as a consequence of the AC load.

Following the continuum mechanical model of Westram et al. [27], during an AC load cycle inhomogeneous domain switching around the crack tip is accompanied by a temporary decrease of the remanent strain perpendicular to the crack in a specimen which is poled perpendicular to the crack. The so induced remanent strain mismatch causes additional tensile stresses at the crack tip, which may contribute to the crack tip load. As mentioned in Section 1, a small region of enhanced electric field and electric displacement in front of the crack tip is expected, although the crack is considered macroscopically permeable. In our experiments, the drop in the fracture toughness already appears for applied nominal fields less than $100 \mathrm{~V} / \mathrm{mm}$ $\left(<0.2 E_{\mathrm{c}}\right)$, so that a fivefold increase of the electric field in front of the crack tip would be necessary to induce local repolarization. According to the ferroelastic tougheningmechanism described in Section 0 , it is expected that also in unpoled specimens a ferroelastic switching zone is generated around the crack tip as a consequence of the applied mechanical load. The remanent strain perpendicular to the crack at the crack tip is then similar to that of a poled sample. This means that the switching model of Westram et al. could explain the increase of $\Delta k_{\mathrm{AC}}$ with increasing field amplitude as a consequence of higher tensile stresses in front of the crack tip. Yet, the observed increase of $\Delta k_{\mathrm{AC}}$ with increasing frequency is not straightforward. Many experiments show that the extent of domain switching decreases with growing frequency or slew rate of the applied electric field [37-40]. With a synchrotron X-ray beam Pojprapai et al. [40] scanned an area of $3.5 \times 2.5 \mathrm{~mm}$ around the crack tip in an edge-notched rectangular sample with a pre-crack that had been driven by an AC electric field. They found that the zone size of preferred domain orientation decreased with increasing frequency. Consequently, $\Delta k_{\mathrm{AC}}$ should decrease with increasing frequency, which is in contradiction to our results.

A possible explanation of the growing toughness reduction with increasing frequency incorporates the fact that the number of electrical load cycles applied until crack initiation increases with the frequency as a consequence of the constant measuring speed. If each AC loading cycle causes inhomogeneous strains between the grains due to piezoelectricity or domain switching, the material around the crack tip will be more fatigued and thus easier to fracture. With a two-dimensional multi-grain model Haug et al. [41] studied the stresses inside grains and across grain boundaries induced by domain switching under electrical loading. An applied field of $4 E_{c}$ gave both tensile and compressive local stresses of up to $+4.5 \sigma_{c}$ and $-3 \sigma_{c}$ concentrated at the grain boundaries $\left(\sigma_{c}\right.$ - coercive stress for mechanical compressive loading). In regions were switching stresses and stresses due to the applied load add to large tensile stresses, micro fracture along the grain boundaries may occur and induce mechanical fatigue crack growth. MacLaren et al. [42] calculated stresses across domain walls from diffraction patterns measured with Transmission Electron Microscopy. They supposed that these stresses could lead to the 
nucleation of microcracks during electrical cycling, which would facilitate transgranular fracture along domain walls. Our images from the crack path and the fracture surface (Figure 6 and Figure 7) show inter- and transgranular fracture for both pure mechanical and combined electromechanical loading. A larger percentage of transgranular fracture during the AC electromechanical loading is not apparent. Due to the low electric load amplitudes as well as the high permittivity of the crack the microscopic events responsible for the effect of the AC electrical loading are assumed to occur only in a small region in the vicinity of the crack much smaller than the ferroelastic switching zone. The conclusions drawn about the mechanism for crack advance are schematically shown in Figure 8.

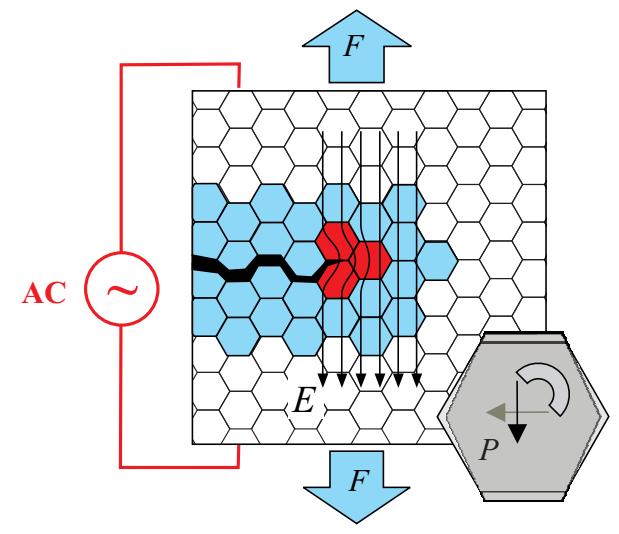

Figure 8 Schematic illustration of an electromechanically loaded crack (AC, F). The electric field $\mathrm{E}$ around the crack is indicated by the black arrows. The zone of ferroelastic switching is marked by the blue (light grey) grains. The small zone around the crack tip, where the alternating electric field could lead to strain mismatch between the single grains due to the inhomogeneous piezoelectric effect and/or ferroelectric switching with polarization reversal, is illustrated by the red (dark grey) grains. The two overlapping enlarged grains, where in each case the overall polarization direction $\mathrm{P}$ is illustrated by an arrow, schematically illustrate the change of the shape of a grain induced by $90^{\circ}$ domain switching.

\section{Conclusions}

R-curve measurements were done with ferroelectric PZT SENB-specimens with additional electrical loads perpendicular to the crack faces. The results can be summarized as follows:

- A DC voltage of $\approx 1 / 3 E_{\mathrm{c}}$ does not influence the critical mechanical loads for crack advance.

- An AC electric field with an amplitude $\approx 1 / 3 E_{\text {c }}$ and a frequency of $1 \mathrm{kHz}$ reduces the fracture toughness of $1 \mathrm{MPa} \sqrt{\mathrm{m}}$ by approximately $0.25 \mathrm{MPa} \sqrt{\mathrm{m}}$, i.e. by approximately $25 \%$.

- For AC loading the relative drop in fracture toughness increases with the applied electric field amplitude and frequency (keeping the same speed of measuring).

- For an unpoled sample an additional AC load yields the same relative drop in the fracture toughness as for a poled sample.

- For unipolar cyclic electrical loading in polarization direction, $\Delta k_{\text {uni, }}$ is much smaller than $\Delta k_{\mathrm{AC}}$. For unipolar electrical loading against the poling direction there is no visible drop in the fracture toughness.

To explain the drop in the fracture toughness caused by AC loads below $1 / 3 E_{\mathrm{c}}$, it is assumed that intergranular stresses, which are induced by the piezoelectric effect or by ferroelectric domain switching, lead to mechanical fatigue crack growth along the grain boundaries. Supposed that the microscopic events causing the drop in the fracture toughness are confined to a small region in front of the crack tip where the electric field is increased, a macroscopic continuum mechanical approach cannot represent the described processes. Instead, a detailed micromechanical model that accounts for the grains and domains of the ceramic is needed to support our assumptions. Such models are under way, but not available yet. We could not experimentally prove domain switching around the crack tip in Piezoresponse-ForceMicroscopy experiments.

So far, it was known that an AC field with an amplitude higher than $E_{\mathrm{c}}$ applied perpendicular to a crack in a ferroelectric material drives the crack, and that a small additional mechanical load leads to higher crack growth rates [28]. Our work confirms that small DC loads have no influence on the fracture toughness, and it provides additional experimental results which show, to which extent the critical mechanical loads are reduced by a small AC load with an amplitude less than $1 / 3 E_{\mathrm{c}}$. The theoretical explanation and the experimental visualization of the underlying mechanisms is the topic of future works.

\section{Acknowledgements}

We thank the DFG (German Science Foundation) for supporting this project under the Grant number SCHN 372/12-2 and BA 1411/12-2. The technical support by Dieter Schmidt is very much appreciated.

\section{Appendix A - Effect of electrical load on mode I crack tip load}

Due to the experimental set-up, a non-homogeneous macroscopic electric field is induced by the applied electric potential. In combination with the piezoelectric properties of the poled samples, this may induce mechanical stresses, even in the mechanically unloaded sample. This circumstance may lead to a macroscopic effect of the electrical load on the mode I crack tip load. Thereby, the term macroscopic describes the effect or the properties arising from the homogenized ceramic, without distinguishing individual grains.

With the help of a 2D plane strain finite element (FE) analysis utilizing the standard linear piezoelectric material model, the relation between applied voltage and the so induced mode I crack tip load is investigated. A sample of $4 \mathrm{~mm}$ height, $23 \mathrm{~mm}$ length and a $2 \mathrm{~mm}$ gap between the Ushaped electrodes is considered. Taking advantage of the symmetric set-up, only one half of the specimen is modelled with the crack in the axis of symmetry. The crack is assumed to be electrically permeable. Hence, the electric potential is set to zero along the entire axis of symmetry, both along the ligament and along the crack faces. Along the ligament the mechanical displacement perpendicular to the ligament is set to zero. A constant electric potential is applied along the U- 
shaped electrodes. The remaining edges are defined free of surface charges. No mechanical tractions are applied along the edges of the model. The material constants of PIC 151 are taken from Kemmer [43].

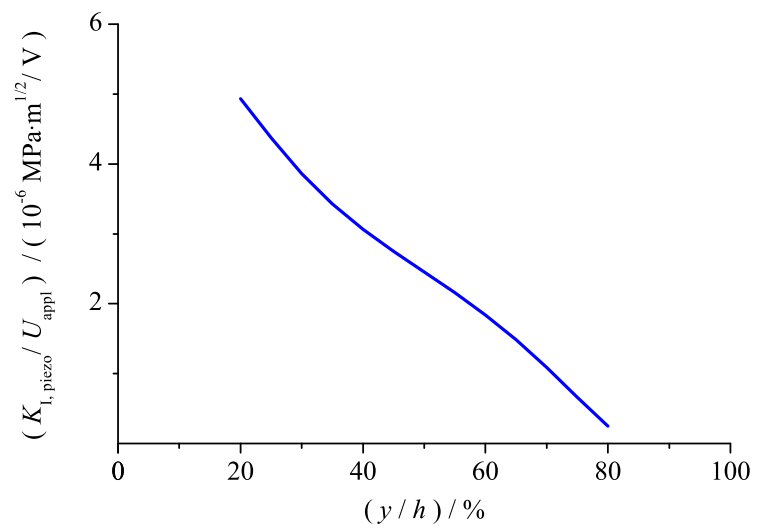

Figure 9 Normalized mode I stress intensity factor over the relative specimen height, showing the contribution of the electrical load on the mode I crack tip load due to piezoelectric coupling.

Due to linearity, the resulting $K_{\mathrm{I}}$ can be related to a nominal electric voltage of $1 \mathrm{~V}$. Thus, $0.5 \mathrm{~V}$ is applied at the modelled electrode causing an electric field against the poling direction. $K_{\mathrm{I}}$ is derived at various different crack lengths between $20 \%<a / h 80 \%$ by stress extrapolation as described by Jelitto et al. [18].

From the results shown in Figure 9, it can be seen that the contribution to $K_{\mathrm{I}}$ due to the macroscopic piezoelectric effect is about $0.0035 \mathrm{MPa} \sqrt{\mathrm{m}}$ at the maximum applied voltage of $700 \mathrm{~V}$ and a relative crack length of $20 \%$. It can be regarded as negligible compared to the fracture toughness reduction of about $0.25 \mathrm{MPa} \sqrt{ } \mathrm{m}$ which is observed under AC-loading with $700 \mathrm{~V}$ magnitude. This conclusion is confirmed by the supplementary measurements using an unpoled sample, which shows the same drop in the fracture toughness under AC-loading as the poled samples.

\section{Appendix B - Mode I crack tip load due to applied forces}

The mode I stress intensity factor $K_{\mathrm{I}}$ caused by the applied forces $F$ can be computed with the help of the nominal bending stress $\sigma$ and the shape function $Y$ using

$$
K=\sigma \sqrt{\pi a} Y(\alpha)
$$

where $\sigma$ and $Y$ are given for a SENB specimen in the literature (for instance by Munz and Fett [36, p. 279]) as:

$$
\begin{gathered}
\sigma=\frac{3 F\left(l_{1}-l_{2}\right)}{2 b h^{2}}, \\
Y(\alpha)=\frac{1.1215}{(1-\alpha)^{3 / 2}}\left[\begin{array}{l}
\frac{5}{8}-\frac{5}{12} \alpha+\frac{1}{8} \alpha^{2}+5 \alpha^{2}(1-\alpha)^{6} \ldots \\
+\frac{3}{8} \exp \left(-6.1342 \frac{\alpha}{(1-\alpha)}\right)
\end{array}\right] .
\end{gathered}
$$

Here, $\alpha$ denotes the relative crack length $a / h, l_{1}$ the outer and $l_{2}$ the inner roller spans, $b$ the sample width and $h$ the sample height.

\section{Appendix C - Nominal electric field}

The electric field in the plane of cracking between the Ushaped electrodes is computed, depending on the coordinate $0<y<h$ over the specimen height. 2D simulations are carried out, taking advantage of the geometric symmetry. With account of the results found in Appendix A, the piezoelectric coupling is neglected within the computations. As a further simplification, the anisotropy of the dielectric constants is considered small and thus neglected. Again the crack is assumed permeable. Finally, a purely linear dielectric analysis is performed. As a consequence of the nature of the electrical load, the electric field distribution does not depend on the actual dielectric constant of the material. The electric potential is set to zero along the plane of cracking and an electric potential (half the applied electric voltage $U$ between the electrodes) larger than zero is applied at the modelled electrode. Again, the remaining edges are set free of surface charges.

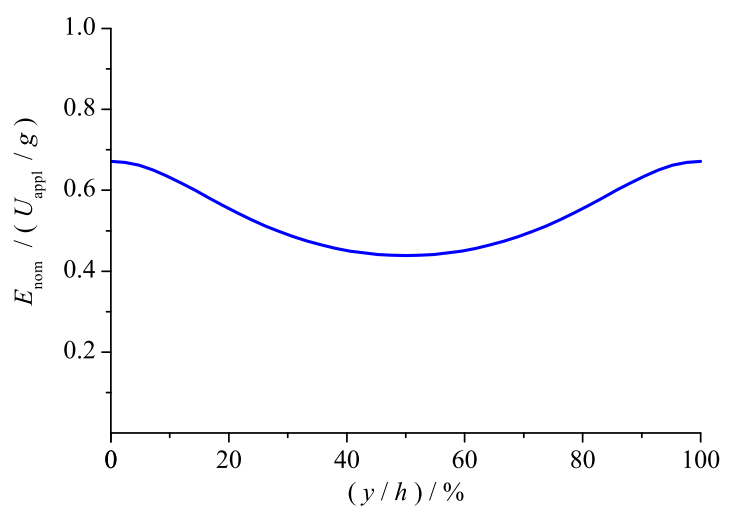

Figure $10 \quad$ Nominal electric field normalized by the applied electrical load across the relative specimen height, for a SENB sample with U-shaped electrodes and a height of $4 \mathrm{~mm}$ and a gap size of $2 \mathrm{~mm}$.

Figure 10 shows the nodal solution of $E_{\text {nom }}$ normalized by $U / g$ over the specimen height for a specimen height $h=4 \mathrm{~mm}$ and a gap between the electrodes $g=2 \mathrm{~mm}$. Due to symmetry, the electric field is perpendicular to the plane of cracking. It is important to notice that the used normalization does not give the correct relation for varying $g$. Therefore, the computation is repeated for every specimen with other $h$ and $g$. For the evaluation of the experimental results the discrete data points are fitted by a polynomial of sixth order. The nominal electric field at the crack tip is then defined as $E_{\text {nom }}=E_{\text {nom }}(y=a)$.

\section{References}

[1] PICeramic, Piezoelectric Ceramic Products. PICeramic $\mathrm{GmbH}$, Lederhose, Germany, 2009, online available from: www.piceramic.de/pdf/KATALOG_english.pdf.

[2] dos Santos e Lucato S.L., Lupascu D.C. \& Rödel J., Effect of poling direction on R-curve behavior in lead zirconate titanate. J. Am. Ceram. Soc., 2000, 83(2), 424-426.

[3] dos Santos e Lucato S.L., Crack-growth-velocitydependent R-curve behavior in lead zirconate titanate. J. Am. Ceram. Soc., 2003, 86(6), 1037-1039. 
[4] Karastamatis T., Lupascu D.C., dos Santos e Lucato S.L., Rödel J. \& Lynch C.S., R-curves of lead zirconate titanate (PZT). J. European Ceram. Soc., 2003, 23(9), 1401-1408.

[5] Jones J.L. \& Hoffman M., R-curve and stress-strain behavior of ferroelastic ceramics. J. Am. Ceram. Soc., 2006, 89(12), 3721-3727.

[6] Kounga Njiwa A.B., Fett T., Lupascu D.C. \& Rödel J., Effect of geometry and electrical boundary conditions on R-curves for lead zirconate titanate ceramics. Eng. Fract. Mech., 2006, 73(3), 309-317.

[7] Meschke F., Kolleck A. \& Schneider G.A., R-curve behaviour of BaTiO3 due to stress-induced ferroelastic domain switching. J. European Ceram. Soc., 1997, 17(9), 1143-1149.

[8] Schneider G.A. \& Heyer V., Influence of the electric field on Vickers indentation crack growth in BaTiO3. J. European Ceram. Soc., 1999, 19(6-7), 1299-1306.

[9] Chen W., Lupascu D.C., Rödel J. \& Lynch C.S., Short crack R-curves in ferroelectric and electrostrictive PLZT. J. Am. Ceram. Soc., 2001, 84(3), 593-597.

[10] Schneider G.A., Influence of electric field and mechanical stresses on the fracture of ferroelectrics. Annual Review of Materials Research, 2007, 37, 491-538.

[11] Fett T., Glazounov A., Hoffmann M.J., Munz D. \& Thun G., On the interpretation of different R-curves for soft PZT. Eng. Fract. Mech., 2001, 68(10), 1207-1218.

[12] Hao T.H. \& Shen Z.Y., A New Electric BoundaryCondition of Electric Fracture-Mechanics and Its Applications. Eng. Fract. Mech., 1994, 47(6), 793-802.

[13] Balke H., Kemmer G. \& Drescher J., Some remarks on the fracture mechanics of piezoelectric solids. Conference Proceedings: Michel B. and Winkler T. (ed.), MicroMaterials Conference "MicroMat 1997", pp. 398 - 401, 1997.

[14] McMeeking R.M., The energy release rate for a Griffith crack in a piezoelectric material. Eng. Fract. Mech., 2004, 71(7-8), 1149-1163.

[15] Landis C.M., Energetically consistent boundary conditions for electromechanical fracture. International Journal of Solids and Structures, 2004, 41(22-23), 6291-6315.

[16] Landis C.M., Energetically consistent boundary conditions for electromechanical fracture (Erratum, vol 41, pg $6291,2004)$. International Journal of Solids and Structures, 2005, 42(8), 2461-2463.

[17] Li W.Y., McMeeking R.M. \& Landis C.M., On the crack face boundary conditions in electromechanical fracture and an experimental protocol for determining energy release rates. Eur. J. Mech. A-Solids, 2008, 27(3), 285-301.

[18] Jelitto H., Felten F., Häusler C., Kessler H., Balke H. \& Schneider G.A., Measurement of energy release rates for cracks in PZT under electromechanical loads. J. European Ceram. Soc., 2005, 25(12), 2817-2820.

[19] Jelitto H., Kessler H., Schneider G.A. \& Balke H., Fracture behavior of poled piezoelectric PZT under mechanical and electrical loads. J. European Ceram. Soc., 2005, 25(5), 749-757.

[20] Häusler C., Jelitto H., Neumeister P., Balke H. \& Schneider G.A., Interfacial fracture of piezoelectric multilayer actuators under mechanical and electrical loading. Int. J. Fract., 2009, 160(1), 43-54.

[21] Schneider G.A., Felten F. \& McMeeking R.M., The electrical potential difference across cracks in PZT measured by Kelvin Probe Microscopy and the implications for fracture. Acta Mater., 2003, 51(8), 2235-2241.

[22] Tobin A.G. \& Pak Y.E., Effect of Electric-Fields on Fracture-Behavior of Pzt Ceramics. Smart Materials - Smart Structures and Materials 1993, 1993, 1916, 78-86.

[23] Cao H.C. \& Evans A.G., Electric-Field-Induced FatigueCrack Growth in Piezoelectrics. J. Am. Ceram. Soc., 1994, 77(7), 1783-1786.

[24] Lynch C.S., Chen L., Suo Z., McMeeking R.M. \& Yang W., Crack-Growth in Ferroelectric Ceramics Driven by Cyclic Polarization Switching. J. Intell. Mater. Syst. Struct., 1995, 6(2), 191-198.

[25] Weitzing H., Schneider G.A., Steffens J., Hammer M. \& Hoffmann M.J., Cyclic fatigue due to electric loading in ferroelectric ceramics. J. European Ceram. Soc., 1999, 19(6-7), 1333-1337.

[26] Shieh J., Huber J.E. \& Fleck N.A., Fatigue crack growth in ferroelectrics under electrical loading. J. European Ceram. Soc., 2006, 26(1-2), 95-109.

[27] Westram I., Oates W.S., Lupascu D.C., Rödel J. \& Lynch C.S., Mechanism of electric fatigue crack growth in lead zirconate titanate. Acta Mater., 2007, 55(1), 301-312.

[28] Westram I., Ricoeur A., Emrich A., Rödel J. \& Kuna M., Fatigue crack growth law for ferroelectrics under cyclic electrical and combined electromechanical loading. J. European Ceram. Soc., 2007, 27(6), 2485-2494.

[29] Nam B.G., Tsuchida S. \& Watanabe K., Fatigue crack growth driven by electric fields in piezoelectric ceramics and its governing fracture parameters. Int. J. Eng. Sci., 2008, 46(5), 397410.

[30] Helke G. \& Kirsch W., Dielektrische und piezoelektrische Eigenschaften der ternären keramischen festen Lösungen von $\mathrm{Pb}(\mathrm{Ni} 1 / 3 \mathrm{Sb} 2 / 3) \mathrm{O} 3-\mathrm{PbTiO} 3-\mathrm{PbZrO} 3$. Hemsdorfer Technische Mitteilungen, 1971, 32, 1010-1015.

[31] Lupascu D.C., Aulbach E. \& Rödel J., Mixed electromechanical fatigue in lead zirconate titanate. Journal of Applied Physics, 2003, 93(9), 5551-5556.

[32] PICeramic, Piezoceramic Materials. PICeramic GmbH, Lederhose, Germany, 2009, online available from: http://www.piceramic.de/pdf/piezo_material.pdf.

[33] Nishida T., Hanaki Y. \& Pezzotti G., Effect of NotchRoot Radius on the Fracture Toughness of a Fine-Grained Alumina. J. Am. Ceram. Soc., 1994, 77(2), 606-608.

[34] Kübler J.J., Bestimmung der Bruchzähigkeit keramischerWerkstoffe mit der SEVNB Methode: Resultate eines VAMAS/ESIS Ringversuches. Conference Proceedings: Muster W., Ziebs J. and Link R. (ed.), Werkstoffwoche, vol. 10, pp. 17-22, Wiley-VCH Weinheim, 1999, 1998.

[35] Jelitto H., Felten F., Swain M.V., Balke H. \& Schneider G.A., Measurement of the total energy release rate for cracks in PZT under combined mechanical and electrical loading. J. Appl. Mech.-Trans. ASME, 2007, 74(6), 1197-1211.

[36] Munz D. \& Fett T. Ceramics - Mechanical Properties, Failure Behaviour, Materials Selection. book series: Springer Series in Materials Science, vol. 36, 2 ed., Springer-Verlag, Berlin, 2001.

[37] Viehland D. \& Chen Y.H., Random-field model for ferroelectric domain dynamics and polarization reversal. Journal of Applied Physics, 2000, 88(11), 6696-6707.

[38] Grossmann M., Bolten D., Lohse O., Boettger U., Waser R. \& Tiedke S., Correlation between switching and fatigue in PbZr0.3Ti0.7O3 thin films. Appl. Phys. Lett., 2000, 77(12), 18941896.

[39] Gruverman A., Wu D. \& Scott J.F., Piezoresponse force microscopy studies of switching behavior of ferroelectric capacitors on a 100-ns time scale. Physical Review Letters, 2008, 100(9), 097601.

[40] Pojprapai S., Russell J., Man H., Jones J.L., Daniels J.E. \& Hoffman M., Frequency effects on fatigue crack growth and crack tip domain-switching behavior in a lead zirconate titanate ceramic. Acta Mater., 2009, 57(13), 3932-3940.

[41] Haug A., Onck P.R. \& Van der Giessen E., Development of inter- and intragranular stresses during switching of ferroelectric polycrystals. International Journal of Solids and Structures, 2007, 44(6), 2066-2078.

[42] MacLaren I., Schmitt L.A., Fuess H., Kungl H. \& Hoffmann M.J., Experimental measurement of stress at a fourdomain junction in lead zirconate titanate. Journal of Applied Physics, 2005, 97(9), 094102.

[43] Kemmer G. Berechnung von elektromechanischen Intensitätsparametern bei Rissen in Piezokeramiken. book series: Fortschritt-Berichte VDI, vol. Reihe 18, Nr. 261, VDI Verlag, Düsseldorf, 2000. 